PROCEEDINGS OF THE

AMERICAN MATHEMATICAL SOCIETY

Volume 131, Number 2, Pages 467-477

S 0002-9939(02)06548-6

Article electronically published on May 17, 2002

\title{
ENDPOINT ESTIMATES FOR CERTAIN COMMUTATORS OF FRACTIONAL AND SINGULAR INTEGRALS
}

\author{
SHANZHEN LU AND QIANG WU
}

(Communicated by Andreas Seeger)

\begin{abstract}
In this paper, the authors obtain the endpoint estimates for a class of non-standard commutators with higher order remainders and their variants. Moreover, the authors show that these operators are actually not bounded in certain cases.
\end{abstract}

\section{IntRoduction AND MAIN RESULTS}

During the development of Calderón-Zygmund operators and their commutators, a class of non-standard singular integrals and commutators with higher order remainders were well studied. There are many works on these topics; see [1], [2], [4], 6], etc. In this paper, we study the non-standard commutator defined by

$$
T_{\alpha}^{A} f(x)=\int_{\mathbb{R}^{n}} \frac{\Omega(x-y)}{|x-y|^{n-\alpha+m-1}} R_{m}(A ; x, y) f(y) d y,
$$

where $0 \leq \alpha<n, \Omega \in \operatorname{Lip}_{1}\left(S^{n-1}\right)$ is homogeneous of degree zero, $m \in \mathbb{N}, A$ has derivatives of order $m-1$ in $\operatorname{BMO}\left(\mathbb{R}^{n}\right)$ and

$$
R_{m}(A ; x, y)=A(x)-\sum_{|\gamma|<m} \frac{1}{\gamma !} D^{\gamma} A(y)(x-y)^{\gamma} .
$$

Here and in what follows, for any locally integrable function $f$ on $\mathbb{R}^{n}$, FeffermanStein's sharp function of $f$ is defined by

$$
f^{\sharp}(x)=\sup _{B \subset \mathbb{R}^{n}} \frac{1}{|B|} \int_{B}\left|f(y)-m_{B}(f)\right| d y,
$$

where $B$ is any ball centered at $x$ and $m_{B}(f)=|B|^{-1} \int_{B} f(z) d z$. Moreover, $f$ is said to belong to $\operatorname{BMO}\left(\mathbb{R}^{n}\right)$ if $f^{\sharp} \in L^{\infty}\left(\mathbb{R}^{n}\right)$ and define $\|f\|_{\text {BMO }}=\left\|f^{\sharp}\right\|_{\infty}$. A well-known property of $\mathrm{BMO}\left(\mathbb{R}^{n}\right)$ is that it is the dual space of the Hardy space $H^{1}\left(\mathbb{R}^{n}\right)$. When $\alpha=0, T_{\alpha}^{A}$ is bounded on $L^{p}\left(\mathbb{R}^{n}\right)$ if $1<p<\infty$ and $\Omega$ satisfies the additional moment conditions

$$
\int_{S^{n-1}} \Omega(x) x^{\gamma} d \sigma(x)=0 \text { for all }|\gamma|=m-1,
$$

Received by the editors May 2, 2001 and, in revised form, September 12, 2001.

2000 Mathematics Subject Classification. Primary 42B20; Secondary 47B38, 47A30, 42B30, 42B35.

Key words and phrases. Commutator, Hardy space, BMO, atom.

This project was supported by the National 973 Foundation of China.

(C)2002 American Mathematical Society 
and when $0<\alpha<n$, it maps $L^{p}\left(\mathbb{R}^{n}\right)$ continuously into $L^{q}\left(\mathbb{R}^{n}\right)$ if $1<p, q<\infty$ and $1 / q=1 / p-\alpha / n$ since $\Omega \in L^{\infty}\left(S^{n-1}\right)$; see [4] and [9], respectively. In this paper, we will study the boundedness properties of these kinds of commutators for the extreme values of $p$. In what follows, to avoid distinguishing the case $\alpha=0$ and the case $0<\alpha<n$ and to simplify the statements, we will constantly use a general boundedness assumption of $T_{\alpha}^{A}$ and let $n / \alpha=\infty$ when $\alpha=0$.

Note that when $m=1, T_{\alpha}^{A}$ degenerates into the classical commutator of the fractional or singular integral

$$
T_{\alpha} f(x)=\int_{\mathbb{R}^{n}} \frac{\Omega(x-y)}{|x-y|^{n-\alpha}} f(y) d y
$$

with the $\operatorname{BMO}\left(\mathbb{R}^{n}\right)$ function $A$. It is shown in [5] that, in general, this commutator does not map $H^{1}\left(\mathbb{R}^{n}\right)$ into $L^{n /(n-\alpha)}\left(\mathbb{R}^{n}\right)$ and $L^{n / \alpha}\left(\mathbb{R}^{n}\right)$ into $\operatorname{BMO}\left(\mathbb{R}^{n}\right)$. However, this is not the case when $m \geq 2$.

Theorem 1. Let $m \geq 2, \Omega \in \operatorname{Lip}_{1}\left(S^{n-1}\right)$ and assume that $A$ has derivatives of order $m-1$ in $\mathrm{BMO}\left(\mathbb{R}^{n}\right)$. If $1<p, q<\infty, \frac{1}{q}=\frac{1}{p}-\frac{\alpha}{n}$ and $T_{\alpha}^{A}$ maps $L^{p}\left(\mathbb{R}^{n}\right)$ continuously into $L^{q}\left(\mathbb{R}^{n}\right)$, then $T_{\alpha}^{A}$ maps $L^{n / \alpha}\left(\mathbb{R}^{n}\right)$ continuously into $\operatorname{BMO}\left(\mathbb{R}^{n}\right)$.

We also consider the variant of $T_{\alpha}^{A}$, which is defined by

$$
\bar{T}_{\alpha}^{A} f(x)=\int_{\mathbb{R}^{n}} \frac{\Omega(x-y)}{|x-y|^{n-\alpha+m-1}} Q_{m}(A ; x, y) f(y) d y
$$

with

$$
Q_{m}(A ; x, y)=R_{m-1}(A ; x, y)-\sum_{|\gamma|=m-1} \frac{1}{\gamma !} D^{\gamma} A(x)(x-y)^{\gamma} .
$$

$\bar{T}_{\alpha}^{A}$ is closely related to $T_{\alpha}^{A}$ since

$$
\bar{T}_{\alpha}^{A} f(x)=T_{\alpha}^{A} f(x)-\sum_{|\gamma|=m-1} \frac{1}{\gamma !}\left[D^{\gamma} A, T_{\alpha, \gamma}\right] f(x),
$$

where $T_{\alpha, \gamma}$ is the singular or fractional integral with the kernel

$$
K_{\alpha, \gamma}(x, y)=\frac{\Omega(x-y)(x-y)^{\gamma}}{|x-y|^{n-\alpha+m-1}}
$$

and for any suitable functions $b$ and $f$ and any suitable linear operator $T$,

$$
[b, T] f(x)=b(x) T f(x)-T(b f)(x) .
$$

By this, we see that if $1<p, q<\infty$ and $1 / q=1 / p-\alpha / n$, the $\left(L^{p}, L^{q}\right)$-boundedness of $T_{\alpha}^{A}$ and $\bar{T}_{\alpha}^{A}$ is almost equivalent. Unlike the classical commutators, $\bar{T}_{\alpha}^{A}$ has a better property on $H^{1}\left(\mathbb{R}^{n}\right)$.

Theorem 2. Let $m \geq 2, \Omega \in \operatorname{Lip}_{1}\left(S^{n-1}\right)$ and assume that $A$ has derivatives of order $m-1$ in $\operatorname{BMO}\left(\mathbb{R}^{n}\right)$. If $1<p, q<\infty, \frac{1}{q}=\frac{1}{p}-\frac{\alpha}{n}$ and $\bar{T}_{\alpha}^{A}$ maps $L^{p}\left(\mathbb{R}^{n}\right)$ continuously into $L^{q}\left(\mathbb{R}^{n}\right)$, then $\bar{T}_{\alpha}^{A}$ maps $H^{1}\left(\mathbb{R}^{n}\right)$ continuously into $L^{n /(n-\alpha)}\left(\mathbb{R}^{n}\right)$.

Theorems 1 and 2 indicate the non-standard commutators with higher order remainders. Their variants have better properties than the classical commutators, although they are more like commutators than the classical Calderón-Zygmund operators. As it is well known that the classical Calderón-Zygmund operators are both $\left(H^{1}, L^{n /(n-\alpha)}\right)$ bounded and $\left(L^{n / \alpha}, \mathrm{BMO}\right)$ bounded. But this is not true for the non-standard commutators and their variants. In fact, by Theorems 1 and 2 , 
the equality (1.2) and the unboundedness properties of classical commutators for the extreme values of $p$, we may expect that, in general, $T_{\alpha}^{A}$ does not map $H^{1}\left(\mathbb{R}^{n}\right)$ into $L^{n /(n-\alpha)}\left(\mathbb{R}^{n}\right)$ and $\bar{T}_{\alpha}^{A}$ does not map $L^{n / \alpha}\left(\mathbb{R}^{n}\right)$ into $\operatorname{BMO}\left(\mathbb{R}^{n}\right)$. This is indeed true. To state our results, we need the concept of an atom. A function $a$ is called an $H^{1}$ atom if there exists a ball $B \subset \mathbb{R}^{n}$ such that $a$ is supported on $B,\|a\|_{\infty} \leq|B|^{-1}$ and $\int a(x) d x=0$. It is well known that the Hardy space $H^{1}\left(\mathbb{R}^{n}\right)$ has the atomic decomposition characterization; see [8, Chapter 3] for details.

Theorem 3. Let $m \geq 2, \Omega \in \operatorname{Lip}_{1}\left(S^{n-1}\right)$ and assume that $A$ has derivatives of order $m-1$ in $\mathrm{BMO}\left(\mathbb{R}^{n}\right)$. If $1<p, q<\infty, \frac{1}{q}=\frac{1}{p}-\frac{\alpha}{n}$ and $T_{\alpha}^{A}$ maps $L^{p}\left(\mathbb{R}^{n}\right)$ continuously into $L^{q}\left(\mathbb{R}^{n}\right)$, then the following two statements are equivalent:

(i) $T_{\alpha}^{A}$ maps $H^{1}\left(\mathbb{R}^{n}\right)$ continuously into $L^{n /(n-\alpha)}\left(\mathbb{R}^{n}\right)$;

(ii) for any $H^{1}$ atom a supported on certain ball $B$ and $u \in 3 B \backslash 2 B$, there is

$$
\int_{(4 B)^{c}}\left|\sum_{|\gamma|=m-1} \frac{1}{\gamma !} K_{\alpha, \gamma}(x, u) \int_{B} D^{\gamma} A(y) a(y) d y\right|^{n /(n-\alpha)} d x \leq C .
$$

Theorem 4. Let $m \geq 2, \Omega \in \operatorname{Lip}_{1}\left(S^{n-1}\right)$ and assume that $A$ has derivatives of order $m-1$ in $\operatorname{BMO}\left(\mathbb{R}^{n}\right)$. If $1<p, q<\infty, \frac{1}{q}=\frac{1}{p}-\frac{\alpha}{n}$ and $\bar{T}_{\alpha}^{A}$ maps $L^{p}\left(\mathbb{R}^{n}\right)$ continuously into $L^{q}\left(\mathbb{R}^{n}\right)$, then the following two statements are equivalent:

(i) $\bar{T}_{\alpha}^{A}$ maps $L^{n / \alpha}\left(\mathbb{R}^{n}\right)$ continuously into $\mathrm{BMO}\left(\mathbb{R}^{n}\right)$;

(ii) for any ball $B$ and $u \in 3 B \backslash 2 B$, there is

$$
\begin{aligned}
\frac{1}{|B|} \int_{B} \mid \sum_{|\gamma|=m-1} \frac{1}{\gamma !}\left[D^{\gamma} A(x)-m_{B}\left(D^{\gamma} A\right)\right] \\
\quad \times \int_{(4 B)^{c}} K_{\alpha, \gamma}(u, y) f(y) d y \mid d x \leq C\|f\|_{n / \alpha} .
\end{aligned}
$$

We remark that Theorems 1-4 are still true if the homogeneous kernels of the form $\frac{\Omega(x-y)}{|x-y|^{n-\alpha+m-1}}$ are replaced by the non-homogeneous kernels $K(x, y)$ satisfying

$$
K(x, y) \leq C|x-y|^{-(n-\alpha+m-1)}
$$

and

$$
\left|\nabla_{x} K(x, y)\right|+\left|\nabla_{y} K(x, y)\right| \leq C|x-y|^{-(n-\alpha+m)} .
$$

However, when these operators have homogeneous kernels, we can obtain a more significant result.

Theorem 5. Let $m \geq 2$, let $\Omega \in \operatorname{Lip}_{1}\left(S^{n-1}\right)$ not be zero, and, if $\alpha=0$, let $\Omega$ satisfy the additional moment conditions (1.1). Suppose that $A$ has derivatives of order $m-1$ in $\mathrm{BMO}\left(\mathbb{R}^{n}\right)$. Then the following three statements are equivalent:

(i) $T_{\alpha}^{A}$ maps $H^{1}\left(\mathbb{R}^{n}\right)$ continuously into $L^{n /(n-\alpha)}$;

(ii) $\bar{T}_{\alpha}^{A}$ maps $L^{n / \alpha}\left(\mathbb{R}^{n}\right)$ continuously into $\mathrm{BMO}\left(\mathbb{R}^{n}\right)$;

(iii) $A$ is a polynomial of degree no more than $m-1$.

It should be noted that the higher order derivatives of $A$ are needed to be in $\operatorname{BMO}\left(\mathbb{R}^{n}\right)$ for our results to be true. This assumption may probably not be removed since there is no criterion on the boundedness of these non-standard commutators analogous with the well-known Coifman-Rochberg-Weiss' theorem for the classical commutators. 
By Theorem 5, one can easily deduce that $T_{\alpha}^{A}$ is not $\left(H^{1}, L^{n /(n-\alpha)}\right)$ bounded and $\bar{T}_{\alpha}^{A}$ is not $\left(L^{n / \alpha}\right.$, BMO) bounded unless $T_{\alpha}^{A}=\bar{T}_{\alpha}^{A}=0$. This conclusion follows from the fact that if $A$ is a polynomial of degree no more than $m-1$, there is $R_{m}(A ; x, y)=Q_{m}(A ; x, y)=0$. However, we remark that although $T_{\alpha}^{A}$ is not $\left(H^{1}, L^{n /(n-\alpha)}\right)$ bounded, we can prove that it maps $H^{1}\left(\mathbb{R}^{n}\right)$ continuously into weak $L^{n /(n-\alpha)}\left(\mathbb{R}^{n}\right)$. In fact, in their recent paper [3], Chen and $\mathrm{Hu}$ have proved this for the case $\alpha=0$. The proof for the case $0<\alpha<n$ is much similar. We will not give the details here.

\section{Proof of the theorems}

We will prove the theorems in this section. We remark that Theorem 1 can be proved by a standard sharp estimate. As for Theorem 2 , because it has been essentially proved in [7] for the case $\alpha=0$ and in [9] for the case $0<\alpha<n$, we will omit its proof. The ideas to prove Theorems 3 and 4 mainly come from [5]. However, the proof of Theorem 5 is not so trivial as that of the classical commutators in [5]. Now let us turn to the proof of the Theorems. We start with a key lemma.

Lemma 2.1 (see [4]). Let $b(x)$ be a function on $\mathbb{R}^{n}$ with $m$-th order derivatives in $L_{\mathrm{loc}}^{q}\left(\mathbb{R}^{n}\right)$ for some $q>n$. Then

$$
\left|R_{m}(b ; x, y)\right| \leq C_{m, n}|x-y|^{m} \sum_{|\alpha|=m}\left(\frac{1}{|\tilde{Q}(x, y)|} \int_{\tilde{Q}(x, y)}\left|D^{\alpha} b(z)\right|^{q} d z\right)^{1 / q}
$$

where $\tilde{Q}(x, y)$ is the cube centered at $x$ and having diameter $5 \sqrt{n}|x-y|$.

Proof of Theorem 1. Noting that

$$
f^{\sharp}(x) \leq 2 \sup _{B \subset \mathbb{R}^{n}} \inf _{c \in \mathbb{R}} \frac{1}{|B|} \int_{B}|f(y)-c| d y
$$

with the supremum taken over all balls centered at $x$ on $\mathbb{R}^{n}$, we need only show that there exists $c_{B}$ so that

$$
\frac{1}{|B|} \int_{B}\left|T_{\alpha}^{A} f(y)-c_{B}\right| d y \leq C\left(\left\|T_{\alpha}^{A}\right\|_{(p, q)}+\sum_{|\gamma|=m-1}\left\|D^{\gamma} A\right\|_{\mathrm{BMO}}\right)\|f\|_{n / \alpha}
$$

holds for any ball $B=B(x, r)$ on $\mathbb{R}^{n}$ with $C$ independent of $B$ and $f$. To do this, write $f_{1}=f \chi_{4 B}$ and $f_{2}=f-f_{1}$, choose $y_{0} \in 3 B \backslash 2 B$, and take $c_{B}=T_{\alpha}^{A} f_{2}\left(y_{0}\right)$. Write

$$
\begin{aligned}
\frac{1}{|B|} \int_{B}\left|T_{\alpha}^{A} f(y)-T_{\alpha}^{A} f_{2}\left(y_{0}\right)\right| d y \leq \frac{1}{|B|} \int_{B}\left|T_{\alpha}^{A} f_{1}(y)\right| d y \\
+\frac{1}{|B|} \int_{B}\left|T_{\alpha}^{A} f_{2}(y)-T_{\alpha}^{A} f_{2}\left(y_{0}\right)\right| d y \equiv I_{1}+I_{2} .
\end{aligned}
$$

Take $1<p<n / \alpha$ and $q$ such that $1 / q=1 / p-\alpha / n$. By the $\left(L^{p}, L^{q}\right)$ boundedness of $T_{\alpha}^{A}$, the term $I_{1}$ can be well estimated:

$I_{1} \leq\left(\frac{1}{|B|} \int_{B}\left|T_{\alpha}^{A} f_{1}(y)\right|^{q} d y\right)^{1 / q} \leq C|B|^{-1 / q}\left\|T_{\alpha}^{A}\right\|_{(p, q)}\left\|f_{1}\right\|_{p} \leq C\left\|T_{\alpha}^{A}\right\|_{(p, q)}\left\|f_{1}\right\|_{n / \alpha}$

To estimate the term $I_{2}$, let

$$
\tilde{A}(y)=A(y)-\sum_{|\gamma|=m-1} \frac{1}{\gamma !} m_{B}\left(D^{\gamma} A\right) y^{\gamma}
$$


Obviously there is $R_{m}(A ; y, z)=R_{m}(\tilde{A} ; y, z)$. By the formula (see [1])

$$
R_{m}(A ; x, y)-R_{m}(A ; x, z)=\sum_{|\beta|<m} \frac{1}{\beta !} R_{m-|\beta|}\left(D^{\beta} A ; z, y\right)(x-z)^{\beta}
$$

It follows from Lemma 2.1 that when $|y-x|<r$ and $2^{k} r<|z-x| \leq 2^{k+1} r$ with $k \geq 2$,

$$
\begin{aligned}
& \left|\frac{\Omega(y-z)}{|y-z|^{n-\alpha+m-1}} R_{m}(\tilde{A} ; y, z)-\frac{\Omega\left(y_{0}-z\right)}{\left|y_{0}-z\right|^{n-\alpha+m-1}} R_{m}\left(\tilde{A} ; y_{0}, z\right)\right| \\
& \leq\left|\frac{\Omega(y-z)}{|y-z|^{n-\alpha+m-1}}-\frac{\Omega\left(y_{0}-z\right)}{\left|y_{0}-z\right|^{n-\alpha+m-1}}\right|\left|R_{m-1}(\tilde{A} ; y, z)\right| \\
& \quad+\left|\frac{\Omega\left(y_{0}-z\right)}{\left|y_{0}-z\right|^{n-\alpha+m-1}}\right|\left|R_{m-1}(\tilde{A} ; y, z)-R_{m-1}\left(\tilde{A} ; y_{0}, z\right)\right| \\
& \quad+\sum_{|\gamma|=m-1}\left|D^{\gamma} \tilde{A}(z)\right|\left|\frac{\Omega(y-z)(y-z)^{\gamma}}{|y-z|^{n-\alpha+m-1}}-\frac{\Omega\left(y_{0}-z\right)\left(y_{0}-z\right)^{\gamma}}{\left|y_{0}-z\right|^{n-\alpha+m-1}}\right| \\
& \leq C \sum_{|\gamma|=m-1}|| D^{\gamma} A \|_{\mathrm{BMO}}|k| y-y_{0}|| y-\left.z\right|^{-(n-\alpha+1)} \\
& \quad+\left|y_{0}-z\right|^{-(n-\alpha+m-1)}\left(\left|y-y_{0}\right|^{m-1}+\sum_{l=1}^{m-2} k\left|y-y_{0}\right|^{l}\left|y_{0}-z\right|^{m-1+l}\right) \\
& \quad+C \sum_{|\gamma|=m-1}\left|D^{\gamma} \tilde{A}(z)\right|\left|y-y_{0}\right||y-z|^{n-\alpha+1} \\
& \leq C \sum_{|\gamma|=m-1}\left(\left\|D^{\gamma} A\right\|_{\mathrm{BMO}}+\left|D^{\gamma} \tilde{A}(z)\right|\right) k 2^{-k}|y-z|^{-(n-\alpha)}
\end{aligned}
$$

where we have omitted some well-known technical computations. Therefore, taking $s, t>1$ so that $1 / s+1 / t+\alpha / n=1$ and using Hölder's inequality, we obtain

$$
\begin{aligned}
& \left|T_{\alpha}^{A} f_{2}(y)-T_{\alpha}^{A} f_{2}\left(y_{0}\right)\right| \\
& \leq \sum_{k=2}^{\infty} \int_{2^{k+1} B \backslash 2^{k} B} \mid \frac{\Omega(y-z)}{|y-z|^{n-\alpha+m-1}} R_{m}(\tilde{A} ; y, z) \\
& \quad-\frac{\Omega\left(y_{0}-z\right)}{\left|y_{0}-z\right|^{n-\alpha+m-1}} Q_{m}\left(\tilde{A} ; y_{0}, z\right) \mid f_{2}(z) d z \\
& \quad \leq C \sum_{k=2}^{\infty} k 2^{-k}\left\|f_{2}\right\|_{n / \alpha}\left(\int_{2^{k+1} B \backslash 2^{k} B}|y-z|^{-(n-\alpha) s} d z\right)^{1 / s} \\
& \quad \times\left(\int_{2^{k+1} B} \sum_{|\gamma|=m-1}\left(\left\|D^{\gamma} A\right\|_{\mathrm{BMO}}+\left|D^{\gamma} \tilde{A}(z)\right|\right)^{t} d z\right)^{1 / t} \\
& \quad \leq C \sum_{|\gamma|=m-1}\left\|D^{\gamma} A\right\|_{\mathrm{BMO}}\left\|f_{2}\right\|_{n / \alpha} \sum_{k=2}^{\infty} k^{2} 2^{-k} \\
& \quad=C \sum_{|\gamma|=m-1}\left\|D^{\gamma} A\right\|_{\mathrm{BMO}}\left\|f_{2}\right\|_{n / \alpha} .
\end{aligned}
$$

Thus,

$$
I_{2} \leq C \sum_{|\gamma|=m-1}\left\|D^{\gamma} A\right\|_{\mathrm{BMO}}\left\|f_{2}\right\|_{n / \alpha} .
$$

Combining the estimate for $I_{1}$ and $I_{2}$, we finish the proof. 
Proof of Theorem 3. Because of the atomic decomposition theory of the space $H^{1}\left(\mathbb{R}^{n}\right)$, a linear operator $T$ being bounded from $H^{1}\left(\mathbb{R}^{n}\right)$ to $L^{q}\left(\mathbb{R}^{n}\right), q \geq 1$, is equivalent to the fact that for any $H^{1}$ atom $a$ there is $\|T a\|_{q} \leq C$. So we need only consider the behavior of $T_{\alpha}^{A}$ acting on an $H^{1}$ atom. Suppose that $a$ is such an atom supported on $B=B\left(x_{0}, r_{0}\right)$. Let

$$
\tilde{A}(x)=A(x)-\sum_{|\gamma|=m-1} \frac{1}{\gamma !} m_{B}\left(D^{\gamma} A\right) x^{\gamma}
$$

then $R_{m}(A ; x, y)=R_{m}(\tilde{A} ; x, y)$. For $u \in 3 B \backslash 2 B$, let

$$
\begin{aligned}
& \mu_{1}(x)=\chi_{4 B}(x) T_{\alpha}^{A} a(x), \\
& \mu_{2}(x, u)=\chi_{(4 B)^{c}}(x) \int_{\mathbb{R}^{n}}\left(\frac{\Omega(x-y)}{|x-y|^{n-\alpha+m-1}} R_{m-1}(\tilde{A} ; x, y)\right. \\
& \left.-\frac{\Omega(x-u)}{|x-u|^{n-\alpha+m-1}} R_{m-1}(\tilde{A} ; x, u)\right) a(y) d y, \\
& \mu_{3}(x, u)=\chi_{(4 B)^{c}}(x) \sum_{|\gamma|=m-1} \frac{1}{\gamma !} \int_{B}\left[K_{\alpha, \gamma}(x, y)-K_{\alpha, \gamma}(x, u)\right] D^{\gamma} \tilde{A}(y) a(y) d y, \\
& \mu_{4}(x, u)=\chi_{(4 B)^{c}}(x) \sum_{|\gamma|=m-1} \frac{1}{\gamma !} \int_{B} K_{\alpha, \gamma}(x, u) D^{\gamma} \tilde{A}(y) a(y) d y .
\end{aligned}
$$

Then by the vanishing condition of $a$, it is not difficult to verify that

$$
T_{\alpha}^{A} a(x)=\mu_{1}(x)+\mu_{2}(x, u)-\mu_{3}(x, u)-\mu_{4}(x, u) .
$$

Taking $n /(n-\alpha)<q<\infty$ and $p$ so that $1 / q=1 / p-\alpha / n$, it follows from the $\left(L^{p}, L^{q}\right)$ boundedness of $T_{\alpha}^{A}$ that

$$
\left\|\mu_{1}\right\|_{n /(n-\alpha)} \leq|4 B|^{(n-\alpha) / n-1 / q}\left\|T_{\alpha}^{A} a\right\|_{q} \leq C|B|^{1-1 / p}\|a\|_{p} \leq C .
$$

In what follows, we assume that $k \geq 2$. When $x \in 2^{k+1} B \backslash 2^{k} B$, using the formula (2.1) and Lemma 2.1 we obtain

$$
\begin{gathered}
\left|\frac{\Omega(x-y)}{|x-y|^{n-\alpha+m-1}} R_{m-1}(\tilde{A} ; x, y)-\frac{\Omega(x-u)}{|x-u|^{n-\alpha+m-1}} R_{m-1}(\tilde{A} ; x, u)\right| \\
\leq\left|\frac{\Omega(x-y)}{|x-y|^{n-\alpha+m-1}}-\frac{\Omega(x-u)}{|x-u|^{n-\alpha+m-1}}\right|\left|R_{m-1}(\tilde{A} ; x, y)\right| \\
\quad+\left|\frac{\Omega(x-u)}{|x-u|^{n-\alpha+m-1}}\right|\left|R_{m-1}(\tilde{A} ; x, y)-R_{m-1}(\tilde{A} ; x, u)\right| \\
\leq C \sum_{|\gamma|=m-1}\left\|D^{\gamma} A\right\|_{\mathrm{BMO}}\left(k|x-y|^{-(n-\alpha+1)}|y-u|\right. \\
\left.\quad+\sum_{l=0}^{m-2}|x-y|^{-(n-\alpha+m-1)+l}|y-u|^{m-1-l}\right) \\
\leq C \sum_{|\gamma|=m-1}\left\|D^{\gamma} A\right\|_{\mathrm{BMO}} k 2^{-k}|x-y|^{-(n-\alpha)} .
\end{gathered}
$$


Thus, we have

$$
\begin{aligned}
\left\|\mu_{2}(\cdot, u)\right\|_{n /(n-\alpha)} & \leq C \sum_{|\gamma|=m-1}\left\|D^{\gamma} A\right\|_{\text {BMO }} \sum_{k=2}^{\infty} k 2^{-k} \\
& \times\left[\int_{2^{k+1} \backslash 2^{k} B}\left(\int_{B}|x-y|^{-(n-\alpha)}|a(y)| d y\right)^{n /(n-\alpha)} d x\right]^{(n-\alpha) / n} \\
\leq & C \sum_{|\gamma|=m-1}\left\|D^{\gamma} A\right\|_{\text {BMO }} \sum_{k=2}^{\infty} k 2^{-k} \\
= & C \sum_{|\gamma|=m-1}\left\|D^{\gamma} A\right\|_{\text {BMO }} .
\end{aligned}
$$

Concerning the term $\mu_{3}(x, u)$, since $\Omega$ is Lipschitz, we obtain

$$
\begin{aligned}
\left\|\mu_{3} \quad(\cdot, u)\right\|_{n /(n-\alpha)} & \\
\leq & C \sum_{|\gamma|=m-1} \sum_{k=2}^{\infty} \\
& \times\left[\int_{2^{k+1} B \backslash 2^{k} B}\left(\int_{B} \frac{|y-u|}{|x-y|^{n-\alpha+1}}\left|D^{\gamma} \tilde{A}(y) a(y)\right| d y\right)^{n /(n-\alpha)}\right]^{(n-\alpha) / n} \\
\leq & C \sum_{|\gamma|=m-1}\left\|D^{\gamma} A\right\|_{\mathrm{BMO} \sum_{k=2}^{\infty} 2^{-k}} \\
\leq & C \sum_{|\gamma|=m-1}\left\|D^{\gamma} A\right\|_{\mathrm{BMO}} .
\end{aligned}
$$

Now we see that $\left\|T_{\alpha}^{A} a\right\|_{n /(n-\alpha)} \leq C$ is equivalent to $\left\|\mu_{4}(\cdot, u)\right\|_{n /(n-\alpha)} \leq C$. Using the vanishing condition of $a$, we see the last expression is just (1.3). This finishes the proof.

Proof of Theorem 4. Let $f \in L^{n / \alpha}\left(\mathbb{R}^{n}\right)$ and for any ball $B \subset \mathbb{R}^{n}$, write

$$
f=f_{1}+f_{2}=f \chi_{4 B}+f \chi_{(4 B)^{c}} .
$$

Also, as in the proof of Theorems 1 and 3 , let

$$
\tilde{A}(x)=A(x)-\sum_{|\gamma|=m-1} \frac{1}{\gamma !} m_{B}\left(D^{\gamma} A\right) x^{\gamma} .
$$

For $u \in 3 B \backslash 2 B$, put

$$
\begin{aligned}
& \sigma_{1}(x)=\bar{T}_{\alpha}^{A} f_{1}(x), \\
& \sigma_{2}(x)=\int_{\mathbb{R}^{n}} \frac{\Omega(x-y)}{|x-y|^{n-\alpha+m-1}} R_{m-1}(\tilde{A} ; x, y) f_{2}(y) d y, \\
& \sigma_{3}(x, u)=\sum_{|\gamma|=m-1} \frac{1}{\gamma !}\left[\left(D^{\gamma} A\right)(x)-m_{B}\left(D^{\gamma} A\right)\right]\left(T_{\alpha, \gamma} f_{2}(x)-T_{\alpha, \gamma} f_{2}(u)\right), \\
& \sigma_{4}(x, u)=\sum_{|\gamma|=m-1} \frac{1}{\gamma !}\left[\left(D^{\gamma} A\right)(x)-m_{B}\left(D^{\gamma} A\right)\right] T_{\alpha, \gamma} f_{2}(u) .
\end{aligned}
$$

Then obviously there is

$$
\bar{T}_{\alpha}^{A} f(x)=\sigma_{1}(x)+\sigma_{2}(x)-\sigma_{3}(x, u)-\sigma_{4}(x, u) .
$$

Noting that $m_{B}\left(\sigma_{4}(\cdot, u)\right)=0$, we have

$$
\begin{aligned}
\bar{T}_{\alpha}^{A} f(x)-m_{B}\left(\bar{T}_{\alpha}^{A} f\right) & =\sigma_{1}(x)-m_{B}\left(\sigma_{1}\right)+\left[\sigma_{2}(x)-\sigma_{2}(u)\right] \\
- & m_{B}\left(\left[\sigma_{2}(\cdot)-\sigma_{2}(u)\right]\right)-\sigma_{3}(x, u)+m_{B}\left(\sigma_{3}(\cdot, u)\right)-\sigma_{4}(x, u) .
\end{aligned}
$$


Like the estimate for $I_{1}$ in the proof of Theorem 1 , it follows from the $\left(L^{p}, L^{q}\right)$ boundedness of $\bar{T}_{\alpha}^{A}$ that

$$
\frac{1}{|B|} \int_{B}\left|\sigma_{1}(x)\right| d x \leq C\left\|D^{\gamma} A\right\|_{\mathrm{BMO}}\|f\|_{n / \alpha} .
$$

By the method of estimating the term $I_{2}$ in the proof of Theorem 1, but a little simpler here, we can show

$$
\left|\sigma_{2}(x)-\sigma_{2}(u)\right| \leq C \sum_{|\gamma|=m-1}\left\|D^{\gamma} A\right\|_{\mathrm{BMO}}\|f\|_{n / \alpha} .
$$

Finally, since $\Omega$ is Lipschitz, a standard computation leads to

$$
\frac{1}{|B|} \int_{B}\left|\sigma_{3}(x, u)\right| d x \leq C \sum_{|\gamma|=m-1}\left\|D^{\gamma} A\right\|_{\mathrm{BMO}}\|f\|_{n / \alpha} .
$$

Then integrating in $x$ on $B$ and using the above estimates we obtain the equivalence of the estimate

$$
\frac{1}{|B|} \int_{B}\left|\bar{T}_{\alpha}^{A} f(x)-m_{B}\left(\bar{T}_{\alpha}^{A} f\right)\right| d x \leq C\|f\|_{n / \alpha}
$$

and the estimate

$$
\frac{1}{|B|} \int_{B}\left|\sigma_{4}(x, u)\right| d x \leq C\|f\|_{n / \alpha} .
$$

Since $B$ is arbitrary, we finish the proof.

Proof of Theorem 5. First note that (iii) obviously implies both (i) and (ii) since when $A$ is a polynomial of degree no more than $m-1, T_{\alpha}^{A}=\bar{T}_{\alpha}^{A}=0$.

Now let us consider the converse. As we have pointed out in Section 1, under the assumptions of the theorem, both $T_{\alpha}^{A}$ and $\bar{T}_{\alpha}^{A}$ are bounded from $L^{p}\left(\mathbb{R}^{n}\right)$ to $L^{q}\left(\mathbb{R}^{n}\right)$. Thus, by Theorems 3 and 4 , we need only show that both (1.3) and (1.4) imply (iii).

We first show that (1.3) implies (iii). Let $a$ be any $H^{1}$ atom and

$$
C_{\gamma}=\frac{1}{\gamma !} \int_{B} D^{\gamma} A(y) a(y) d y .
$$

Suppose that $a$ is supported on the ball $B=B\left(x_{0}, r_{0}\right)$. By (1.3), for any $u \in 3 B \backslash 2 B$,

$$
\begin{aligned}
C & \geq \int_{(4 B)^{c}}\left|\sum_{|\gamma|=m-1} C_{\gamma} \frac{\Omega(x-u)(x-u)^{\gamma}}{|x-u|^{n-\alpha+m-1}}\right|^{n /(n-\alpha)} d x \\
& \geq \int_{7 r_{0}<|x-u|<N r_{0}}\left|\sum_{|\gamma|=m-1} C_{\gamma} \frac{\Omega(x-u)(x-u)^{\gamma}}{|x-u|^{n-\alpha+m-1}}\right|^{n /(n-\alpha)} d x \\
& =\int_{7 r_{0}}^{N r_{0}} r^{-1} \int_{S^{n-1}}\left|\sum_{|\gamma|=m-1} C_{\gamma} \Omega(x) x^{\gamma}\right|^{n /(n-\alpha)} d \sigma(x) d r \\
& =\log (N / 7) \int_{S^{n-1}}\left|\sum_{|\gamma|=m-1} C_{\gamma} \Omega(x) x^{\gamma}\right|^{n /(n-\alpha)} d \sigma(x),
\end{aligned}
$$

where $N>7$ is any large positive integer. Noting that $\log (N / 7) \rightarrow \infty$ as $N \rightarrow \infty$, 
we must have

$$
\int_{S^{n-1}}\left|\sum_{|\gamma|=m-1} C_{\gamma} \Omega(x) x^{\gamma}\right|^{n /(n-\alpha)} d \sigma(x)=0
$$

This implies

$$
\sum_{|\gamma|=m-1} C_{\gamma} \Omega(x) x^{\gamma}=0
$$

Since $\Omega$ is not zero, as the result of the fact that $\Omega(x) x^{\gamma},|\gamma|=m-1$, are linear independent, we obtain $C_{\gamma}=0$ for all $\gamma,|\gamma|=m-1$. That is,

$$
\int_{B} D^{\gamma} A(y) a(y) d y=0
$$

Since $a$ is arbitrary, $D^{\gamma} A$ must be constant. This means $A$ must be a polynomial of degree no more than $m-1$.

Let us turn to show (1.4) implies (iii). Let $\left\{\gamma^{i}\right\}_{i=1}^{M}$ be all the multi-indices such that $\left|\gamma^{i}\right|=m-1$. Denote by $U$ the matrix with its coefficients

$$
u_{i j}=u_{j i}=\frac{1}{\gamma^{i} ! \gamma^{j !}} \int_{S^{n-1}}|\Omega(x)|^{2} x^{\gamma^{i}} x^{\gamma^{j}} d \sigma(x)
$$

Since $\Omega(x) x^{\gamma^{i}}, 1 \leq i \leq M$, are linear independent, we have $\operatorname{det}(U) \neq 0$. For any fixed $\gamma,|\gamma|=m-1$, there exists unique $i(\gamma)$ such that $\gamma=\gamma^{i(\gamma)}$. Let $e_{i(\gamma)}$ be the $i(\gamma)$-th unit coordinate basis vector in $\mathbb{R}^{M}$ and put

$$
\left(c_{\gamma, 1}, c_{\gamma, 2}, \cdots, c_{\gamma, M}\right)=e_{i(\gamma)} U^{-1}
$$

Let

$$
g_{\gamma}(x)=\sum_{i=1}^{M} c_{\gamma, i} \frac{1}{\gamma^{i} !} \Omega(x) x^{\gamma^{i}}
$$

Suppose that $B=B\left(x_{0}, r_{0}\right)$ is any fixed ball on $\mathbb{R}^{n}$. Let

$$
h_{\gamma, N}(x)=g_{\gamma}\left(\frac{x}{|x|}\right)|x|^{-\alpha} \chi_{\left\{7 r_{0}<|x|<N r_{0}\right\}}(x)
$$

and for any $u \in 3 B \backslash 2 B$ and any large integer $N>7$, let $f_{\gamma, N}(x)=h_{\gamma, N}(u-y)$. Then $f_{\gamma, N} \in L^{n / \alpha}\left(\mathbb{R}^{n}\right)$ and

$$
\left\|f_{\gamma, N}\right\|_{n / \alpha}=[\log (N / 7)]^{\alpha / n}\left(\int_{S^{n-1}}\left|g_{\gamma}(x)\right|^{n / \alpha} d \sigma(x)\right)^{\alpha / n}
$$


At the same time, we note that the left-hand side of (1.4) is larger than

$$
\begin{aligned}
\frac{1}{|B|} \int_{B}\left|\sum_{j=1}^{M} \frac{1}{\gamma^{j} !}\left[D^{\gamma^{j}} A(x)-m_{B}\left(D^{\gamma^{j}} A\right)\right] \int_{(4 B)^{c}} K_{\alpha, \gamma^{j}}(u, y) f_{\gamma, N}(y) d y\right| d x \\
\geq \frac{1}{|B|} \int_{B} \mid \sum_{j=1}^{M} \frac{1}{\gamma^{j !}}\left[D^{\gamma^{j}} A(x)-m_{B}\left(D^{\gamma^{j}} A\right)\right] \\
\quad \times \int_{7 r_{0}<|y-u|<N r_{0}} \frac{\Omega(u-y)(u-y)^{\gamma^{j}}}{|u-y|^{n-\alpha+m-1}} f_{\gamma, N}(y) d y \mid d x \\
=\frac{1}{|B|} \int_{B} \mid \sum_{j=1}^{M} \frac{1}{\gamma^{j !}}\left[D^{\gamma^{j}} A(x)-m_{B}\left(D^{\gamma^{j}} A\right)\right] \\
\quad \times \sum_{i=1}^{M} c_{\gamma, i} \frac{1}{\gamma^{i !}} \int_{7 r_{0}}^{N r_{0}} r^{-1} \int_{S^{n-1}}|\Omega(x)|^{2} x^{\gamma^{j}} x^{\gamma^{i}} d \sigma(y) d r \mid d x \\
=\log (N / 7) \frac{1}{|B|} \int_{B}\left|\sum_{j=1}^{M}\left[D^{\gamma^{j}} A(x)-m_{B}\left(D^{\gamma^{j}} A\right)\right] \sum_{i=1}^{M} c_{\gamma, i} u_{i j}\right| d x \\
=\log (N / 7) \frac{1}{|B|} \int_{B}\left|D^{\gamma} A(x)-m_{B}\left(D^{\gamma} A\right)\right| d x .
\end{aligned}
$$

Thus, by (1.4), there is

$$
[\log (N / 7)]^{1-\alpha / n} \frac{1}{|B|} \int_{B}\left|D^{\gamma} A(x)-m_{B}\left(D^{\gamma} A\right)\right| d x \leq C .
$$

Letting $N \rightarrow \infty$, we obtain

$$
\frac{1}{|B|} \int_{B}\left|D^{\gamma} A(x)-m_{B}\left(D^{\gamma} A\right)\right| d x=0 .
$$

Since $B$ is arbitrary, $D^{\gamma} A$ must be constant. Hence, $A$ must be a polynomial of degree no more than $m-1$. So far, the proof of Theorem 5 is completed.

\section{ACKNOWLEDGEMENT}

The authors want to express their deep thanks to the referee for his/her several valuable remarks and suggestions.

\section{REFERENCES}

[1] B. Bajsanski and R. Coifman, On singular integrals, Proc. Sympos. Pure Math. Vol 10, Amer. Math. Soc. Providence, R. I. (1967), 1-17. MR 38:6405

[2] A. P. Calderón, On algebras of singular integral operators, Proc. Sympos. Pure Math. Vol 10, Amer. Math. Soc. Providence, R. I. (1967), 18-55. MR 52:15112

[3] W. Chen and G. Hu, Weak type $\left(H^{1}, L^{1}\right)$ estimate for a multilinear singular integral operator, Adv. in Math., 30:1 (2001), 63-69. CMP 2001:12

[4] J. Cohen and J. Gosselin, A BMO estimate for multilinear singular integrals, Illinois J. Math. 30 (1986), 445-464. MR 87k:42022

[5] E. Harboure, C. Segovia and J. L. Torrea, Boundedness of commutators of fractional and singular integrals for the extreme values of $p$, Illinois J. Math. 41 (1997), 676-700. MR 99j: 42025

[6] S. Hofmann, On certain non-standard Calderón-Zygmund operators, Studia Math. 109 (1994), 105-131. MR 95h:42021

[7] G. Hu and D. Yang, Multilinear oscillatory singular integral operators on Hardy spaces, Chinese J. of Contemporary Math. 18 (1997), 403-413. MR 99g:42017 
[8] E. M. Stein, Harmonic Analysis: Real-Variable Methods, Orthogonality, and Oscillatory Integrals, Princeton Univ. Press, Princeton, N. J., 1993. MR 95c:42002

[9] Q. Wu and D. Yang, On fractional multilinear singular integrals, Math. Nachr. to appear.

Department of Mathematics, Beijing Normal University, Beijing 100875, People's Republic of China

E-mail address: lusz@bnu.edu.cn

Department of Mathematics, Beijing Normal University, Beijing 100875, People's Republic of China 